\title{
EDITORIAL
}

\section{A 20 years conundrum of neonatal encephalopathy and hypoxic ischemic encephalopathy: are we closer to a consensus guideline?}

\author{
Pediatric Research (2019) 86:548-549; \\ https://doi.org/10.1038/s41390-019-0547-9
}

The appropriate terminology for neonatal encephalopathy (NE) and hypoxic ischemic encephalopathy (HIE) has been a subject of debate for the last 20 years. ${ }^{1-3}$ Two prior editorials by this journal have covered this conundrum of definitions over the last decade., ${ }^{4,5}$

The last editorial emphasized how the term HIE is misused at birth, as we often do not know when hypoxia ischemia is indeed the cause of NE. The authors recommended instead a descriptive terminology as we do not need to use etiologic labels for disease entities and to avoid obstacles for research by assuming etiology. Understanding the etiology of NE may allow the development of targeted adjunctive therapies related to the underlying mechanism and development of preventative strategies. It is especially important for medicolegal clarity where we might do harm by attributing a cause (hypoxia ischemia) to the disorder (encephalopathy) without having measured cerebral oxygenation and blood flow. ${ }^{5}$

The American College of Obstetricians and the American Academy of Pediatrics (AAP) recommends the HIE diagnosis to include: (1) the presence of a sentinel event occurring immediately before or during labor and delivery for example a ruptured uterus or severe abruption placentae; fetal heart rate monitor patterns consistent with an acute peripartum or intrapartum event; (2) the presence of fetal acidosis and low Apgar scores, (3) evidence of multiple organ injury affecting the heart, liver, or kidneys, (4) neuroimaging with MRI consistent with acute peripartum or intrapartum event and excluding other causes. Before a causal relationship between an acute intrapartum interruption of placental blood flow and later cerebral palsy can be established, the American Congress of Obstetricians and Gynecologists Task Force on Neonatal Encephalopathy and Cerebral Palsy require essential criteria: (1) evidence of a metabolic acidosis in fetal umbilical cord arterial blood obtained at delivery, (2) early onset of $\mathrm{NE}$, (3) cerebral palsy of the spastic quadriplegic or dyskinetic type, and (4) exclusion of other identifiable etiologies such as trauma, coagulation disorders, infectious conditions, or genetic disorders. ${ }^{6}$

The term asphyxia indicates impaired gas exchange leading to progressive hypoxia, hypercarbia, and acidosis depending on the extent and duration of interrupted placental perfusion and gas exchange. The "dose" and duration of hypoxia and the degree of ischemia while well established in animal models, do not have clinically precise clinical biochemical criteria. Not all causes of NE result from HIE. ${ }^{7}$ A diagnosis of HIE or asphyxia is often over-utilized in practice and not clinically justified by the limited data at birth. However an accurate diagnosis of HIE has definitive utility, especially once it is confirmed prior to discharge and after confirmatory tests and neuroimaging are obtained. In many cases, hypoxia ischemia may contribute to NE but is not the prime cause. ${ }^{8}$

This current editorial focus is to provide a descriptive terminology "umbrella" for NE at birth and to limit the HIE diagnosis to infants who meet the full AAP criteria upon discharge from the hospital. A diagnosis of NE does not commit to a specific etiology or pathogenesis and can develop following a constellation of maternal factors, familial factors, genetic predisposition, HIE, infections, placental abnormalities, thrombophilia, coagulation defects, and metabolic disorders. A postulated NE terminology can be proposed at birth such as (1) hypoxic NE, (2) inflammatory NE, (3) infectious $\mathrm{NE}$, (4) metabolic NE. Neuroimaging using magnetic resonance imaging, conventional, diffusion, and spectroscopy gives a useful guide regarding the potential timing of a cerebral insult especially diffusion abnormalities. Abnormalities from a cerebral injury become most evident after 5-7 days using qualitative MRI, and diffusion and spectroscopy are useful to determine the time of the insult. ${ }^{9}$ Blood cultures and placental pathology can identify infectious and inflammatory etiologies. Genetic and metabolic causes can be indicated if a different pattern of brain injury is detected by neuroimaging such as porencephaly, focal arterial infarction, or atypical patterns. Recognizing the etiology of NE involved can also guide investigations such as metabolic and sepsis workups to ensure optimal management.

An accurate HIE diagnosis continues to be helpful to identify parental support such as the HOPE group lead by Mrs Pilon and is highlighted in the family reflection piece in this issue of the journal "Family Reflections: Hope for HIE". ${ }^{10}$ Societies, referrals and parental support groups are built around HIE. A correct diagnosis is important for prognosis, correct identification for enrollment in neuroprotective therapies as well as referral to subspecialties such as occupational therapies and physical therapies and use of appropriate billing.

The words we use make a significant impact on patient care, research and health resources. We hope this editorial brings to light the nuances and differences and why it is important to use distinct terminology for NE and HIE where appropriate.

\section{ADDITIONAL INFORMATION}

Competing interests: The authors declare no competing interests.

Publisher's note: Springer Nature remains neutral with regard to jurisdictional claims in published maps and institutional affiliations.

Lina Chalak ${ }^{1}$, Donna M. Ferriero ${ }^{2}$, Pierre Gressens ${ }^{3}$, Eleanor Molloy ${ }^{4}$ and Cynthia Bearer ${ }^{5}$

${ }^{1}$ UT Southwestern Medical Center of Dallas, Dallas, TX, USA; ${ }^{2}$ Weill Institute for Neurosciences, San Francisco, CA, USA; ${ }^{3}$ Hopital Robert Debre, Paris, France; ${ }^{4}$ University of Dublin Trinity College, Dublin, Ireland and ${ }^{5}$ Department of Pediatrics, University of Maryland, Baltimore, MD, USA

Correspondence: Lina Chalak (lina.chalak@utsouthwestern.edu) 


\section{REFERENCES}

1. Nelson, K. B. et al. Antecedents of neonatal encephalopathy in the Vermont Oxford Network Encephalopathy Registry. Pediatrics 130, 878-886 (2012).

2. Badawi, N. et al. Antepartum risk factors for newborn encephalopathy: the Western Australian case-control study. BMJ 317, 1549-1553 (1998).

3. Volpe, J. J. Neonatal encephalopathy: an inadequate term for hypoxic-ischemic encephalopathy. Ann. Neurol. 72, 156-166 (2012).

4. Molloy, E. J. \& Bearer, C. Neonatal encephalopathy versus hypoxic-ischemic encephalopathy. Pediatr. Res. 84, 574-574 (2018).

5. Dammann, O., Ferriero, D. \& Gressens, P. Neonatal encephalopathy or hypoxicischemic encephalopathy? Appropriate terminology matters. Pediatr. Res. 70, 1-2 (2011).

6. American Academy of Pediatrics, American College of Obstetricians and Gynecologists. Neonatal encephalopathy and cerebral palsy: defining the pathogenesis and pathophysiology. American Academy of Pediatrics; Washington, DC: American College of Obstetricians and Gynecologists, Elk Grove Village, IL (2014).

7. Chalak, L. F., Laptook, A. R., Jafri, H. S., Ramilo, O. \& Perlman, J. M. Clinical chorioamnionitis, elevated cytokines, and brain injury in term infants. Pediatrics 110, 673-680 (2002).

8. Mir, I. N. et al. Placental pathology is associated with severity of neonatal encephalopathy and adverse developmental outcomes following hypothermia. Am. J. Obstet. Gynecol. 213, 849.e1-7 (2015).

9. Cowan, F. et al. Origin and timing of brain lesions in term infants with neonatal encephalopathy. Lancet 361, 736-742 (2003).

10. Pilon, B. Family reflections: hope for HIE. Pediatr. Res. (2019). https://doi.org/ 10.1038/s41390-019-0460-2. [Epub ahead of print]. 